04,06

\title{
Антирезонансные диэлектрические спектры: теория и эксперимент
}

\author{
() А.В. Турик ${ }^{1,2}$, Н.А. Болдырев ${ }^{1}$, Е.И. Ситало ${ }^{1}$, Л.А. Резниченко ${ }^{1}$ \\ ${ }^{1}$ Научно-исследовательский институт фризики Южного федерального университета, \\ Ростов-на-Дону, Россия \\ 2 Южный федеральный университет, \\ Ростов-на-Дону, Россия \\ E-mail: turik1934@yandex.ru
}

(Поступила в Редакцию 20 декабря 2016 г.)

Установлено, что в керамиках твердых растворов мультиферроиков $\mathrm{BiFeO}_{3}-\mathrm{SrTiO}_{3}$ и $\mathrm{BiFeO}_{3}-\mathrm{BaTiO}_{3}$, наряду с релаксационными и резонансными диэлектрическими спектрами, возможен еще один, не описанный в литературе, вид спектров - антирезонансные. Выполнено моделирование антирезонансных диэлектрических спектров и получены экспериментальные данные, подтверждающие результаты моделирования. Показано, что для антирезонансных спектров характерна большая энергия активации $(U>1 \mathrm{eV})$. Предположена связь антирезонансных спектров с явлениями прыжковой проводимости.

Работа выполнена при финансовой поддержке базовой части гос. задания МОН РФ (проекты № 3.6371.2017/БЧ, № 3.6439.2017/БЧ) с использованием оборудования ЦКП „Электромагнитные, электромеханические и тепловые свойства твердых тел“ НИИ физики ЮФУ.

DOI: 10.21883/FTT.2017.07.44590.448

\section{1. Введение}

В керамиках твердых растворов (ТР) на основе мультиферроика феррита висмута $\mathrm{BiFeO}_{3}-\mathrm{SrTiO}_{3}$ и $\mathrm{BiFeO}_{3}-\mathrm{BaTiO}_{3}$ при высоких температурах нами наблюдались отрицательные значения действительной части $\varepsilon^{* \prime}$ комплексной диэлектрической проницаемости $\varepsilon^{*}=\varepsilon^{* \prime}-i \varepsilon^{* \prime \prime}\left(\varepsilon^{* \prime \prime}-\right.$ мнимая часть комплексной диэлектрической проницаемости) в области инфранизких частот. Для объяснения наблюдаемых явлений мы использовали предложенный в [1] подход, основанный на применении эквивалентной схемы замещения керамики параллельно включенными емкостью $C$ и комплексной проводимостью $G=1 / R=\left(G_{1}-i G_{2}\right)$. Такой подход позволяет корректно описать экспериментальные данные, в том числе монотонную зависимость $\varepsilon^{* \prime}$ и $\varepsilon^{* \prime \prime}$ от круговой частоты $\omega=2 \pi \nu$ электрического поля и прохождение действительной части комплексной емкости $C^{*}$ или комплексной диэлектрической проницаемости $\varepsilon^{*}$ керамики через нуль.

\section{2. Теория}

Существование отрицательных проницаемостей $\varepsilon^{\prime}<0$ в металлах и плазме было предсказано в работе [2], но не было измерено вследствие чрезвычайно большого отношения электропроводности к диэлектрической проницаемости. Однако недавно [3,4] вновь возник интерес к механизмам возникновения отрицательной емкости в неупорядоченных твердых телах. Гигантская отрицательная диэлектрическая проницаемость в инфранизкочастотном диапазоне была обнаружена в керамике $\mathrm{CaCu}_{3} \mathrm{Ti}_{4} \mathrm{O}_{12}$ [5]. Авторы [5] предположили, что основным механизмом может быть механизм заполнения и опустошения ловушек. Ниже $10^{-2} \mathrm{~Hz}$ как действительная $\left|\varepsilon^{\prime}\right|$, так и мнимая $\varepsilon^{\prime \prime}$ части проницаемости увеличивались при уменьшении частоты $v$, и $\left|\varepsilon^{\prime}\right|$ быстро достигала величин $\left|\varepsilon^{\prime}\right|>10^{6}$ на частоте $v \approx 10^{-4} \mathrm{~Hz}$. При этом действительная часть удельной проводимости $\gamma^{\prime} \approx$ const. Согласно [1], такое поведение можно промоделировать в терминах цепи конденсатор-резистор $[3,4]$.

Захваченные на ловушки с различной глубиной залегания носители заряда должны для дальнейшего движения преодолевать потенциальные барьеры различной высоты $U$. Керамика является гетерогенной средой и может быть охарактеризована эффектом запаздывания, который описывается формулой Аррениуса [6]

$$
\tau=\tau_{0} \exp \left(\frac{U}{k T}\right)
$$

Здесь $\tau-$ время релаксации, $U-$ средняя высота потенциального барьера, $T$ - абсолютная температура, $k$ - постоянная Больцмана и $\tau_{0}$ - предэкспоненциальный фактор, обратно пропорциональный частоте малых колебаний захваченного носителя вблизи локального минимума потенциальной энергии. Дальнейшее движение носителей можно интерпретировать путем введения комплексной подвижности носителей и комплексной проводимости [1].

Параллельное включение емкости и проводимости позволяет адекватно описать экспериментальные данные и прохождение через нуль действительной части комплексной емкости $C^{*}$ и проницаемости $\varepsilon^{*}$. В отличие от гомогенных материалов, характеризующихся действительными величинами емкости и проводимости, емкость и проводимость гетерогенных материалов комплексны и имеют как действительные, так и мнимые части. 
Следуя [1], мы будем использовать параллельную цепь с действительной емкостью $C$ и комплексной проводимостью $G=1 / R=\left(G_{1}-i G_{2}\right)$. Комплексный адмитанс $Y^{*}$ и комплексная емкость $C^{*}$ такой цепи равны

$$
Y^{*}=\frac{1}{R}+i \omega C=G_{1}-i G_{2}+i \omega C, \quad C^{*}=C-\frac{G_{2}}{\omega}-i \frac{G_{1}}{\omega}
$$

соответственно. При этом действительная $\varepsilon^{* \prime}$ и мнимая $\varepsilon^{* \prime \prime}$ части диэлектрической проницаемости керамики связаны с мнимой $\gamma^{\prime \prime}$ и действительной $\gamma^{\prime}$ частями удельной проводимости соотношениями

$$
\varepsilon^{* \prime}=\varepsilon^{\prime}-\frac{\gamma^{\prime \prime}}{\omega \varepsilon_{0}}, \quad \varepsilon^{* \prime \prime}=\frac{\gamma^{\prime}}{\omega \varepsilon_{0}},
$$

где $\varepsilon_{0}=8.854 \cdot 10^{-12} \mathrm{~F} / \mathrm{m}$ - проницаемость вакуума. (Здесь и в дальнейшем символами со звездочкой нами обозначены параметры гетерогенной среды - керамики, а символами без звездочки - параметры гомогенных компонентов эквивалентной схемы замещения).

\section{3. Основные результаты и обсуждение}

На рис. 1 и 2 приведены экспериментально полученные с помощью универсального измерительного моста Novocontrol ALPHA High-Resolution Dielectric Analyzer и рассчитанные по приведенным выше формулам диэлектрические спектры исследуемых объектов. Керамики ТР $\mathrm{BiFeO}_{3}-\mathrm{SrTiO}_{3}$ и $\mathrm{BiFeO}_{3}-\mathrm{BaTiO}_{3}$ синтезировались методом твердофазных реакций обжигом в две стадии с промежуточным помолом [7]. Подгонка экспериментальных данных с помощью формул (2) и (3) для параллельной $R C$-цепи вполне удовлетворительна. Гиперболическая зависимость $\varepsilon^{* \prime}$ и $\varepsilon^{* \prime \prime}$ от частоты $\omega-$ следствие прыжковой проводимости. Причем для возникновения антирезонанса с отрицательной величиной $\varepsilon^{* \prime}$ достаточно, чтобы диэлектрик имел нормальную величи-

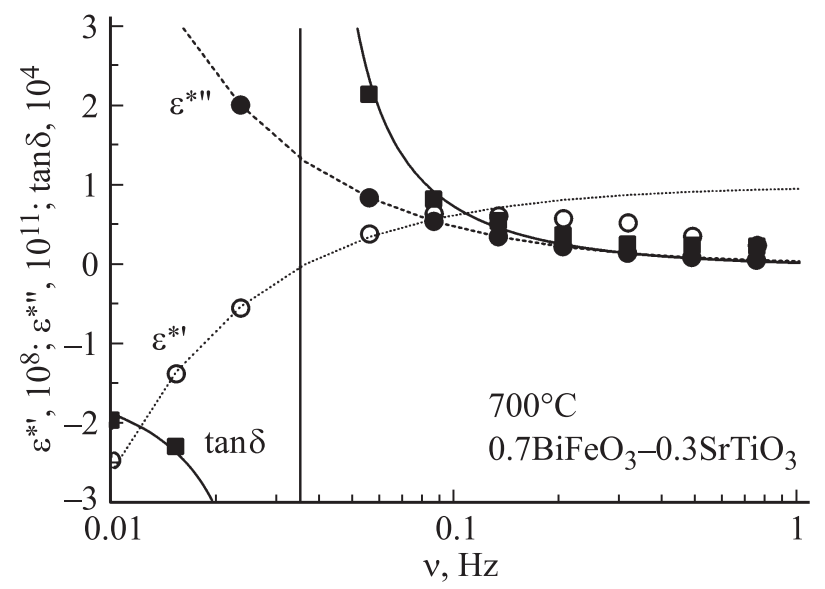

Рис. 1. Полученные экспериментально и рассчитанные по формулам (2) и (3) диэлектрические спектры ТР $0.7 \mathrm{BiFeO}_{3}-0.3 \mathrm{SrTiO}_{3}$ при температуре $700^{\circ} \mathrm{C} . \quad \varepsilon^{\prime}=10^{8}$, $\gamma^{\prime}=0.22(\Omega \cdot \mathrm{m})^{-1}, \gamma^{\prime \prime}=2 \cdot 10^{-4}(\Omega \cdot \mathrm{m})^{-1}$.

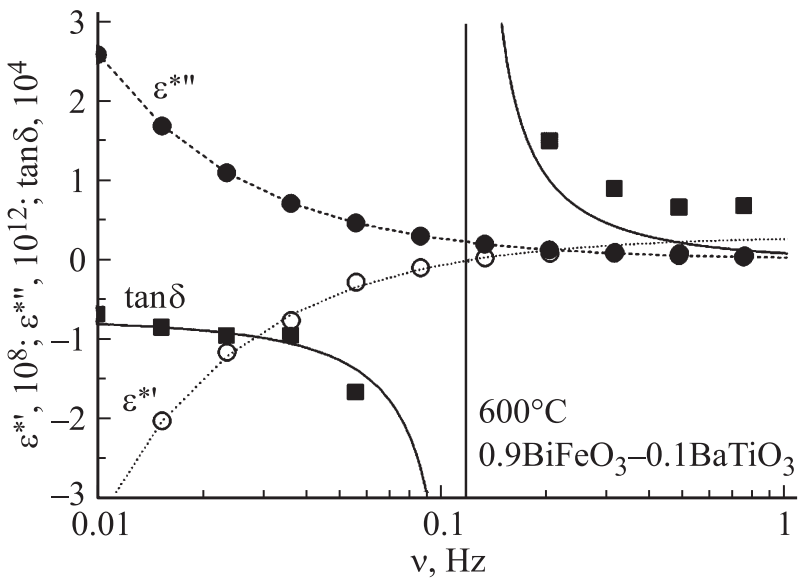

Рис. 2. Полученные экспериментально и рассчитанные по формулам (2) и (3) диэлектрические спектры ТР $0.9 \mathrm{BiFeO}_{3}-0.1 \mathrm{BaTiO}_{3}$ при температуре $600^{\circ} \mathrm{C} . \varepsilon^{\prime}=0.3 \cdot 10^{8}$, $\gamma^{\prime}=1.45(\Omega \cdot \mathrm{m})^{-1}, \gamma^{\prime \prime}=2.17 \cdot 10^{-4}(\Omega \cdot \mathrm{m})^{-1}$.

ну $\varepsilon^{\prime}$, большую $\gamma^{\prime}$ и малую $\gamma^{\prime \prime}$. Критическая частота, на которой $\varepsilon^{* \prime}=0$, определяется как $\omega_{c}=2 \pi v_{c}=\gamma^{\prime \prime} / \varepsilon_{0} \varepsilon^{\prime}$. Частотные зависимости действительной $M^{\prime}(\omega)$ и мнимой $M^{\prime \prime}(\omega)$ частей электрического модуля $M=1 / \varepsilon^{*}$ монотонны, причем $M^{\prime}(\omega)=0$ при $\omega=\omega_{c}$. Для антирезонансных спектров характерна большая $(U>1 \mathrm{eV})$ энергия активации, которую можно оценить по формуле Аррениуса (1). Следует отметить, что большие величины удельной проводимости $\gamma^{\prime}$ характерны не только для резонансных, но и для релаксационных (вследствие максвелл-вагнеровской релаксации) спектров некоторых близких по структуре к исследуемым ТР материалов, таких как керамика $\mathrm{Bi}_{0.5} \mathrm{La}_{0.5} \mathrm{MnO}_{3}$ [7].

\section{4. Заключение}

Установлено теоретически и подтверждено экспериментально, что, наряду с релаксационными и резонансными спектрами диэлектриков, возможен новый, не описанный в литературе, вид спектров - антирезонансные с большой $(U>1 \mathrm{eV})$ энергией активации. Обсуждена связь антирезонансных спектров с явлениями прыжковой проводимости.

\section{Список литературы}

[1] A.V. Turik, A.S. Bogatin. Func. Mater. Lett. 8, 4, 1550035 (2015).

[2] O.V. Dolgov, D.A. Kirzhnits, E.G. Maksimov. Rev. Mod. Phys. 53, 81 (1981).

[3] H.L. Kwok. Solid-State Electron. 47, 1089 (2003).

[4] H.L. Kwok. Phys. Status Solidi C 5, 2, 638 (2008).

[5] Yu. Kabirov, V. Gavrilyachenko, E. Panchenko, E. Milov, A. Klenushkin. Adv. Mater. Res. 705, 52 (2013).

[6] Г. Фрёлих. Теория диэлектриков. ИИЛ, М. (1960). 252 с.

[7] А.В. Турик, А.В. Павленко, Л.А. Резниченко. ФТТ 58, 1499 (2016). 